\title{
Features of molecular-mass distribution of humic acids from permafrost peats of Russian Arctic
}

\author{
Vasilevich R.S. ${ }^{1}$, Vezhov K.S. ${ }^{1}$, Lodygin E.D. ${ }^{1}$, Abakumov E.V. ${ }^{2}$ \\ ${ }^{1}$ Institute of Biology of Komi Science Centre of the Ural Branch of the Russian Academy of \\ Sciences, Syktyvkar, Russia, vasilevich.r.s@ib.komisc.ru \\ ${ }^{2}$ Saint-Petersburg State University, Saint-Petersburg, Russia
}

Keywords: Arctic, permafrost peat mounds, humic acids, molecular-mass distribution

doi: 10.36291/HIT.2019.vasilevich.048

The peatlands investigated are in the ecoton south tundra - forest-tundra (Komi Republic, Russia, Plots 1-1, 1-2) and ecoton north tundra - south tundra (Nenets Autonomous Okrug, Russia, Plots 2-1, 2-2). Humic acids (HAs) from the two permafrost peat soils - Hemic Folic Cryic Histosol (Plot 1-1, Plot 2-1) and Hemic Folic Cryic Histosol (Turbic) (Plot 1-2, Plot 2-2) have been studied from peat plateau-thermokarst complexes. A quantitative assessment of the content of functional groups and molecular fragments of studied HAs $\left({ }^{13} \mathrm{C}\right.$-NMR CP/MAS data) indicates the difference in their molecular composition in seasonal thawed (STL) and permafrost layers $(\mathrm{PL})$ of peat. Specifically, the aromatic fragments and methoxy groups show increasing trends and paraffin groups show decreasing trends with respect to depth for all soils. The obtained values of the number average molecular weights $\left(M_{n}\right)$ of the studied HA preparations have a significant variation $(6.9-23.1 \mathrm{kDa})$ in peat profiles (Fig.). The highest $M_{n}$ of HAs are confined to the upper horizons of the STL, due to the greater contribution of long chain paraffin structures (up to $35 \%$ ) and polysaccharide fragments (up to $27 \%$ ), which leads to an increase in the Stokes radius of HA molecules. Increasing the degree of peat decomposition down the profile and the associated greater humification of the organic material, cause a systematic decrease in $M_{n}$. Profile reduction of $M_{n}$ is due to decrease of proportion of high and medium molecular weight fractions and increase of proportion of low molecular weight fraction. The $M_{n}$ increment in the lower horizons of plots 1-1,1-2, 2-2 is likely due to the coagulation of HAs on the mineral matrix of peat deposits (the peat of these horizons has high ash content) to form high molecular weight HAs. Thus, it has been shown that the humification of organic peat material is associated with the transformation of high molecular weight $\mathrm{HAs}$ structures enriched in paraffin and carbohydrate fragments to condensed structures with lower molecular weights.

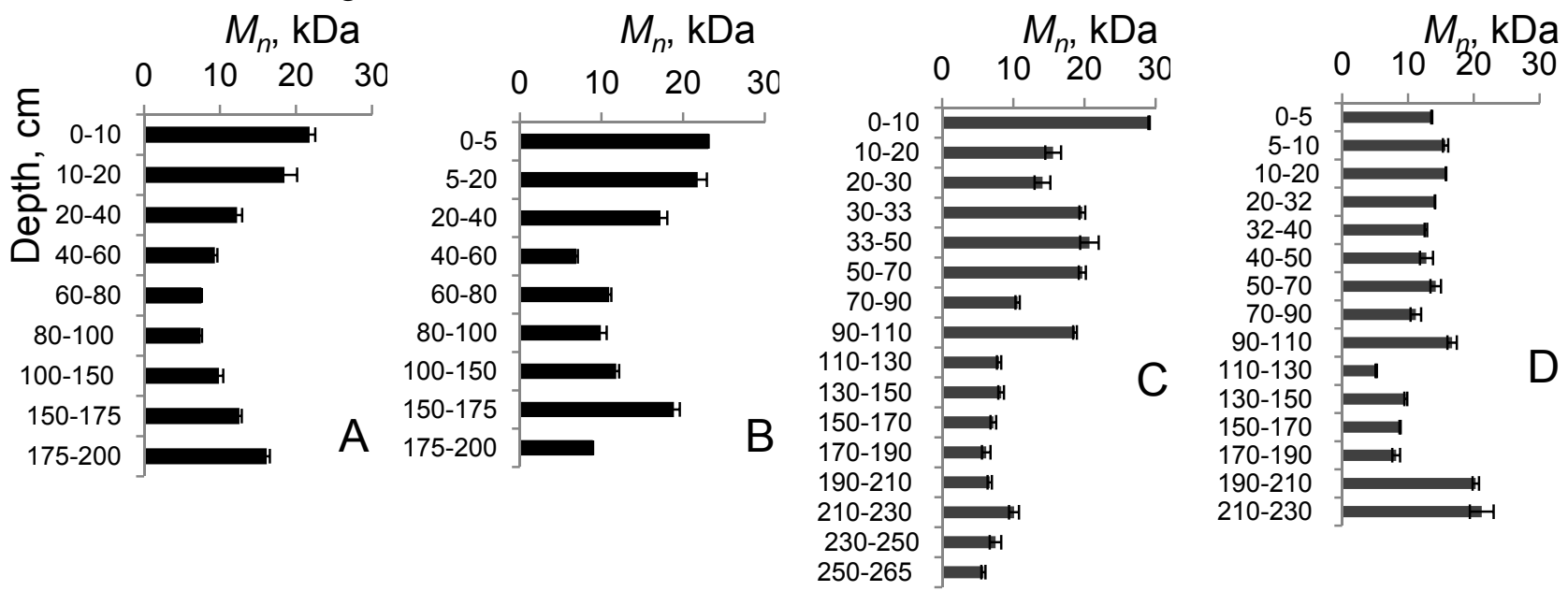

Figure. Number average molecular weight of the HAs from the Plot 1-1 (A), Plot 1-2 (B), Plot 2-1 (C), Plot 2-2 (D).

Acknowledgements. The research was financially supported by the RFBR within the scientific project no. 18-05-60195. 